\title{
VÍTIMAS DE TRAUMA CRÂNIO-ENCEFÁLICO E SEU RETORNO A PRODUTIVIDADE APÓS 6 MESES E 1 ANO.*
}

\author{
HEAD INJURY VICTMS AND THEIR RETURN TO PRODUCTIVITY \\ AFTER 6 MONTHS AND 1 YEAR.
} Regina Marcia Cardoso de Sousa*
Maria Sumie Koizumi**

SOUSA. R.M.C.de; KOIZUMI, M.S. Vítimas de trauma crânio-encefálico e seu retorno a produtividade após 6 meses e 1 ano. Rev.Esc.Enf.USP, v.33, n.3, p. 313-22, set. 1999.

\section{RESUMO}

Trata-se de um estudo prospectivo que analisa o retorno a produtividade de vítimas de trauma crânio-encefálico (TCE) de diferentes gravidades, com idade entre 12 e $6 C$ anos. As vitimas foram examinadas aos 6 meses e 1 ano pós-trauma e seu retomo à produtividade foi analisado, considerando as variáveis nível de escolaridade e tipo de ocupąäo anterior ao trauma. A maioria $(73,6 \%)$ retornou a atividade produtiva aos 6 meses e a freqüencia de retorno à produtividade foi maior 1 ano após TCE. Quanto ao retorno ao tralıalho remunerado e nível de escolaridade ou tipo de ocupação anterior, não houve associação estatística significativa.

UNITERMOS: Traumatismos da cabeça. Recuperação.

\begin{abstract}
A prospective and longitudinal study about head injury victims was carried out in order to identify their return to productivity after 6 months and 1 year post-trauma and to analyze the relationship between their return to work and their educational level as well as to determine the relationship between their return to work and their type of job before the onset of the injury. Of the 72 patients who were students or employees pre-injury, or housewives at the time of injury, the majority $(73,6 \%)$ had returned to productivi $y$ months after onset of injury. Of the victims who had returned to productivity, $25.0 \%$ had changed their original occupations or reported significant problems when returning to productivity. Baseline return was achieved in $48.6 \%$ of the victims at 6 months. Return to productivity was higher at 1 year after injury than at 6 months. When the relationship between return to work and educational level and job type was analyzed, return to work was not related to educational level or job type.
\end{abstract}

UNITERMS: Head injuries. Recovery.

\section{INTRODUÇÃO}

O traumatismo crânio-encefálico (TCE) é uma causa comum de morte e incapacidades, particularmente na primeira metade da vida, sendo apontado como a causa de morte mais freqüente entre 2 e 42 anos de idade ${ }^{15,20,21,26}$. O seu papel dentro dos traumas em geral é relevante quando se observa que em cerca de $50 \%$ dos pacientes politraumatizados há TCE associado e que este tipo de trauma tem se mostrado um grande agravante no prognóstico das vítimas, visto que o sistema nervoso central possui uma grande vulnerabilidade à agressão e a sua capacidade de recuperação é limitada ${ }^{2,5,9,20}$

As vítimas que sobrevivem ao TCE podem apresentar deficiências e incapacidades temporárias ou permanentes, que interferem na capacidade do indivíduo desempenhar funções e cumprir papéis que dele são esperados.

\footnotetext{
* Trabalho baseado em tese de doutoramento apresentada ao Programa Interunidades de Doutoramento EEUSP/EERP. Apresentado em 7 th World Federation of Neurocience Nurses Congress/Plenary session, Amsterdam/The Netherlands, July, 1997.

* Professor Doutor do Departamento de Enfermagem Médico-Cirúrgica da Escola de Enfermagem da Universidade de São Paulo.

*** Professor Titular da Escola de Enfermagem da Universidade de São Paulo.
} 
Uma das principais metas da recuperação consiste em retornar o indivíduo a sua atividade anterior, isto $c$, retorno ao emprego, à escola ou às atividades domésticas ou outra relevante ${ }^{19}$.

Assim considerado, o retorno à produtividade é uma das formas de medida de recuperação de um trauma ou doença e é particularmente relevante quando se examina o ônus individual ou social do trauma, onde a maioria dos atingidos é o adulto jovem.

$\mathrm{Na}$ análise do retorno a produtividade, um outro aspecto a ser considerado e o trabalho remunerado. Ele é tradicionalmente visto na sociedade moderna como proposta central de vida, e assume, papel principal no suprimento tanto das necessidades de auto-estima como econômicas?

O retorno a atividade remunerada é particularmente uma medida relevante quando se examina as vítimas de trauma visando delinear seu ônus social. A perda da própria independência econômica, muitas vezes vinculada a prover recursos a outros elementos da família; a necessidade de destinar verbas para suprir financeiramente a esses indivíduos e a pessoas a eles vinculadas economicamente e o desgaste social pela perda da produtividade de um indivíduo com habilitação para o trabalho, torna de particular interesse a análise do retorno a esse tipo de ocupação.

As conseqüencias da impossibilidade do indivíduo retornar às suas atividades empregatícias, vão além da perda financeira e interferem também na capacidade das vítimas de reconstruir sua vida social. ODDY et al $^{23}$ analisando vítimas de TCE grave, 2 e 7 anos após trauma, observaram que o reajustamento social era acentuadamente restrito aos poucos que trabalhavam. Para os demais, muitos problemas foram encontrados, os quais se assemelhavam aos de uma pessoa desempregada: a perda de uma distribuição de tempo estruturada no cotidiano e de um grupo de amigos; a perda de objetivos para alcançar ou oportunidades para mostrar competência e a ausência de alguma fonte de identidade ou posições.

A capacidade de retornar ao trabalho após o trauma ou doença tem sido relacionada não somente a condições físicas, cognitivas e emocionais mas também a fatores que não são relativos à situação de saúde. Os resultados de outros estudos têm apontado fatores econômicos e pessoais como importantes determinantes do retorno ao trabalho remunerado. Entre esses fatores, a pouca escolaridade e alguns tipos de ocupação têm sido indicados como uma das condições que influenciam negativamente esse retorno ${ }^{11,18,19,24,27}$.

É importante salientar que se distinguem, nos estudos dos fatores relacionados ao retorno ao trabalho remunerado, grupos com diferentes necessidades de atenção para esse retorno e ao mesmo tempo destacam-se certos grupos de individuos que têm particularmente maiores probabilidades de não retornar ao trabalho.

\section{OBJETIVOS}

Caracterizar o retorno à produtividade da vítima de TCE aos 6 meses e 1 ano pós-trauma.

Analisar retorno ao trabalho remunerado e as possíveis associações com o nível de escolaridade e o tipo de ocupação anterior ao trauma.

\section{MATERIAL E MÉTODO}

A população de estudo foi constituída por vítimas de TCE admitidas para tratamento no Instituto Central do HCFMUSP no período de março a junho de 1993, em decorrência de acidente ou violência ocorridos nesse mesmo período.

Os critérios de inclusão das vítimas foram: ter entre 12 e 60 anos de idade; apresentar dados registrados no seu prontuário ou cadastro geral do hospital para sua localização; residir na Grande São Paulo; comparecer ao ambulatório por convocação e consentir em participação da pesquisa.

Excluíram-se aqueles que solicitaram alta a pedido ou evadiram-se do hospital devido à baixa possibilidade de sua aderência a um estudo de seguimento e a possibilidade de diferentes conseqüências do trauma em vítimas que não completaram tratamento na fase aguda pós. traumática. Foram também excluídos vítimas que apresentaram reincidência de trauma, em período inferior a 6 meses.

Os dados das vítimas foram obtidos, inicialmente, em lista fornecida pela Divisão de Arquivo Médico, confirmados pelos dados do prontuário de cada paciente e pelas entrevistas individuais feita por uma das pesquisadoras, durante o período de setembro de 1993 a agosto de 1994.

Da lista da Divisão de Arquivo Médico constaram, além da informação referente ao tipo de TCE, penetrante ou fechado, os seguintes dados: número de registro do paciente no hospital; idade; sexo; óbitos ocorridos na internação; tempo de permanência hospitalar e data de internação e alta.

Nesta lista foram selecionados os pacientes sobreviventes, internados nos meses de março, abril, maio e junho de 1993, das faixas etárias de 12 a 60 anos. Na situação em que a idade era um 
dado não mencionado na lista, o paciente foi mantido na coleta de dados até ser possível a obtenção deste dado, confirmando ou não sua inclusão na população. Os pacientes reinternados só foram incluídos quando sua primeira internação também ocorreu no período especificado.

Feita esta primeira seleção, iniciou-se o processo de busca de prontuários através do número do registro geral dos pacientes no hospital. Localizado o prontuário e confirmados os demais dados de seleção foi realizada convocação para comparecimento ao Ambulatório.

Para cada paciente foi enviada carta solicitando comparecimento ambulatorial, em data marcada entre 6 e 8 meses após a ocorrência do trauma. Neste período era realizada a $1^{a}$ entrevista desde que o paciente concordasse em participar do estado. Neste comparecimento tomou-se o cuidado de verificar a clisponibilidade das vítimas para uma $2^{\mathrm{a}}$ entrevista realizada entre 12 e 14 meses após o trauma, período em que os pacientes também foram notificados através de carta para comparecimento ambulatorial.

Quanto à convocação de pacientes, determinou-se ainda que quando ocorria a falta em primeira convocação uma segunda carta para comparecimento ambulatorial era enviada, tanto na $1^{\mathrm{a}}$ como na $2^{\mathrm{a}}$ entrevista. Nos casos em que não havia comparecimento, mesmo perante as duas correspondências, o paciente era automaticamente eliminado do seguimento pós-traumático. Quando havia menção de número telefônico em alguma das fontes de dados do paciente, este também era um dos meios utilizados para localização das vítimas, em ambas entrevistas. Somente os pacientes internados na época das avaliações eram entrevistados nas instituições e os impossibilitados de comparecer ao Ambulatório, no próprio domicílio.

Produtividade pós-trauma foi definida no presente estudo como retorno ao emprego, escola ou afazeres do lar, considerando-se assim como MORRIS et al $^{22}$ tais atividades de essencial importância para produção nacional à medida que contribuem ou fornecem estrutura para manutenção de sua ocorrência.

Para possibilitar a avaliação do retorno à produtividade pos-trauma, a categorização das vítimas quanto à ocupação principal e à análise da condição ocupacional pós-trauma, fizeram-se necessárias.

Através de dados da primeira entrevista, cada paciente era classificado de acordo com sua ocupação principal. Ocupação principal foi então definida como a mais importante atividade exercida antes do TCE, referida pelo paciente. Devido à dificuldade em estabelecer prioridades entre atividades das vítimas estudantes e com ocupação remunerada, ambas as atividades foram consideradas conjuntamente como ocupação principal quando assim presentes. Dentro dessa classificação estabeleceu-se então as seguintes categorias: ocupação remunerada; estudantes; estudantes que também mantinham ocupação remunerada; aposentados e desempregados.

A condição pós-trauma, em relação à ocupação principal, foi classificada, tendo como base o retorno à produtividade, ou seja, atividade interrompida, alterada devido ao trauma e retorno com o mesmo desempenho.

Os dados estão apresentados em freqüencia absoluta e relativa sendo mostrados em forma de tabelas e figuras. Algumas provas estatísticas também fizeram-se necessárias durante o estudo para complementar a análise dos dados obtidos.

-Prova de associação pelo Qui-quadrado: foi empregada para verificar as eventuais associações entre retorno ao trabalho e tipo de ocupação que as vítimas mantinham anteriormente ao trauma.

-Prova de Fisher: foi empregada ao analisar o retorno ao trabalho e escolaridade e para algumas análises de retorno ao trabalho e tipo de ocupação, uma vez que as condições de aplicação do teste Qui-quadrado não foram satisfeitas em tabela $2 \times 2$.

Todas as provas estatísticas utilizadas foram feitas admitindo um erro de primeira espécie de $5 \%$.

\section{RESULTADOS E DISCUSSÃO}

\section{População Estudada}

Entre março e junho de 1993, 385 vítimas de trauma com diagnóstico de TCE foram admitidas no Instituto Central do HCFMUSP. Destas, $73(19,0 \%)$ morreram antes da alta hospitalar; $65(16,9 \%)$ eram sobreviventes menores de 12 anos e $34(8,8 \%)$ sobreviventes maiores de 60 anos. Portanto, foram 213 as vítimas selecionadas para inclusão no estudo.

Dessas 213 vítimas de TCE, 4 foram excluídas (1 por alta a pedido; 2 evadiram-se do hospital no período agudo de atendimento e 1 por ter reincidência de trauma num período inferior a 6 meses); 5 moravam fora da Grande São Paulo; 38 não apresentavam dados de prontuário ou de cadastro geral que possibilitasse sua localização (umas não tinham residência fixa; outras não possuíam qualquer referência de endereço ou telefone e a maioria apresentava registros de dados incorretos ou incompletos).

Foram, portanto, 166 os indivíduos convocados para a $1^{\text {a }}$ entrevista e destes, foram $83(50,0 \%)$ as vítimas que ao final da coleta de dados pode-se conhecer a evolução de 1 ano pós-trauma. Estes participantes tinham como principais características as descritas a seguir. 
- Apresentar diferentes níveis de gravidade do trauma, estabelecido pelo sistema AIS/ISS ${ }^{*}: 44,6 \%$ trauma leve; $43,4 \%$ moderado e $12 \%$ grave.

- Ter diferentes níveis de alteração de consciência na primeira avaliação feita pela equipe de neurocirurgia: 8,4\% com ECGl (Escala de Coma de Glasgow) $\leq 8$; $15,6 \%$ com ECGl entre 9 e 12; 72,3\% com ECGl $\geq 13$.

- As vítimas tinham idade entre 12 e 60 anos sendo que a maioria, $69,9 \%$, tinha até 35 anos.

\section{Retorno a Produtividade}

Nas 83 vítimas das quais conhecia-se a evolução de 1 ano analisou-se comparativamente seu retorno à produtividade, considerando-se o período de 6 meses e 1 ano. Para tal análise inicialmente essas vítimas foram classificadas segundo sua ocupação principal, sendo apresentada sua distribuição segundo essa categorização na Tabela 1 .

Tabela 1 - Distribuição das vítimas com evolução de 1 ano segundo sua ocupação principal. São Paulo, 1993/94.

\begin{tabular}{|c|c|c|}
\hline Ocupação principal & $\mathbf{n}$ & $\%$ \\
\hline $\begin{array}{l}\text { remunerada } \\
\text { estudante e ocupação remunerada } \\
\text { estudante } \\
\text { desempregado } \\
\text { atividades do lar } \\
\text { aposentado }\end{array}$ & $\begin{array}{l}47 \\
13 \\
09 \\
09 \\
03 \\
02\end{array}$ & $\begin{array}{l}56,7 \\
15,7 \\
10,8 \\
10,8 \\
3,6 \\
2,4\end{array}$ \\
\hline TOTAL & 83 & 100,0 \\
\hline
\end{tabular}

A maioria das vítimas (56,7\%) tinha ocupação remunerada como atividade principal. Além disso, entre os 22 estudantes, a maioria (13) também tinha atividade remunerada. Devido ao critério de inclusão na população de vítimas de até 60 anos, a menor freqüência foi a de aposentados $(2,4 \%)$. O desemprego ocorreu em 10,8\% dos avaliados, sendo tal freqüencia compatível com a taxa de desemprego da região no período. Segundo informações da Fundação Sistema de Análise de Dados (SEADE), a taxa de desemprego na Grande São Paulo, no primeiro semestre de 1993, foi em média de 15,4.

$\mathrm{Na}$ época do evento traumático, $72(86,8 \%)$ pessoas apresentavam atividades ocupacionais e foram analisadas quanto a seu retorno à produtividade tanto aos 6 como aos 12 meses após o trauma. Para subsidiar tal análise, agrupou-se os traumatizados, segundo sua condição em relação a ocupação principal em 3 categorias conforme descrito na metodologia e a seguir discriminadas.

- Atividade interrompida: nesta categoria foram incluídos indivíduos que após o trauma mantinham-se afastados do trabalho remunerado exercido regularmente e estudantes e pessoas envolvidas em afazeres do lar afastadas de suas atividades. Quando o indivíduo mantinha ocupação remunerada e era estudante, só era incluído nessa categoria se afastado das duas atividades.

- Alterada devido ao trauma: foram incluídos nesta categoria indivíduos com mudança de ocupação conseqüente às condições pós-traumáticas; retorno à ocupação anterior sem conseguir o mesmo nível de desempenho; retorno à ocupação anterior reduzindo o tempo dedicado ao seu desempenho; desempregado após trauma, porém mantendo trabalho remunerado exercido de forma irregular; estudantes e pessoas envolvidas em atividades do lar que retornaram a essas atividades sem conseguir manter o mesmo desempenho. Fizeram parte também desse grupo indivíduos que mantinham tanto ocupação remunerada como atividades escolares, caso estivessem afastados de uma dessas duas atividades, ou ainda, se numa delas ou ambas apresentassem alteração de desempenho.

- Retorno com o mesmo desempenho: neste grupo foram incluídos indivíduos que retornaram a sua ocupação principal com o mesmo nível de desempenho.

Para análise comparativa apresenta-se na Tabela 2 as vítimas que mantinham atividades produtivas segundo ocupação principal e sua condição em relação a essa atividade após o trauma, nos 2 diferentes períodos de avaliação.

\footnotetext{
A AIS (Abbreviated Injury Scale) é um sistema avaliativo, de base anatômica que classifica cada lesão decorrente de um trauma, regino corpórea e estabelece um escore que indica gravidade de uma lesao isoladamente. A gravidade global do trauma é obtida pelo ISS (Injury Severity Score) escore matematicamente calculado a partir do AIS ${ }^{1}$.
} 
Tabela 2 - Distribuição das vítimas com atividade produtiva no período anterior ao trauma segundo ocupação principal e condição ocupacional aos 6 e 12 meses após TCE. São Paulo, 1993/94.

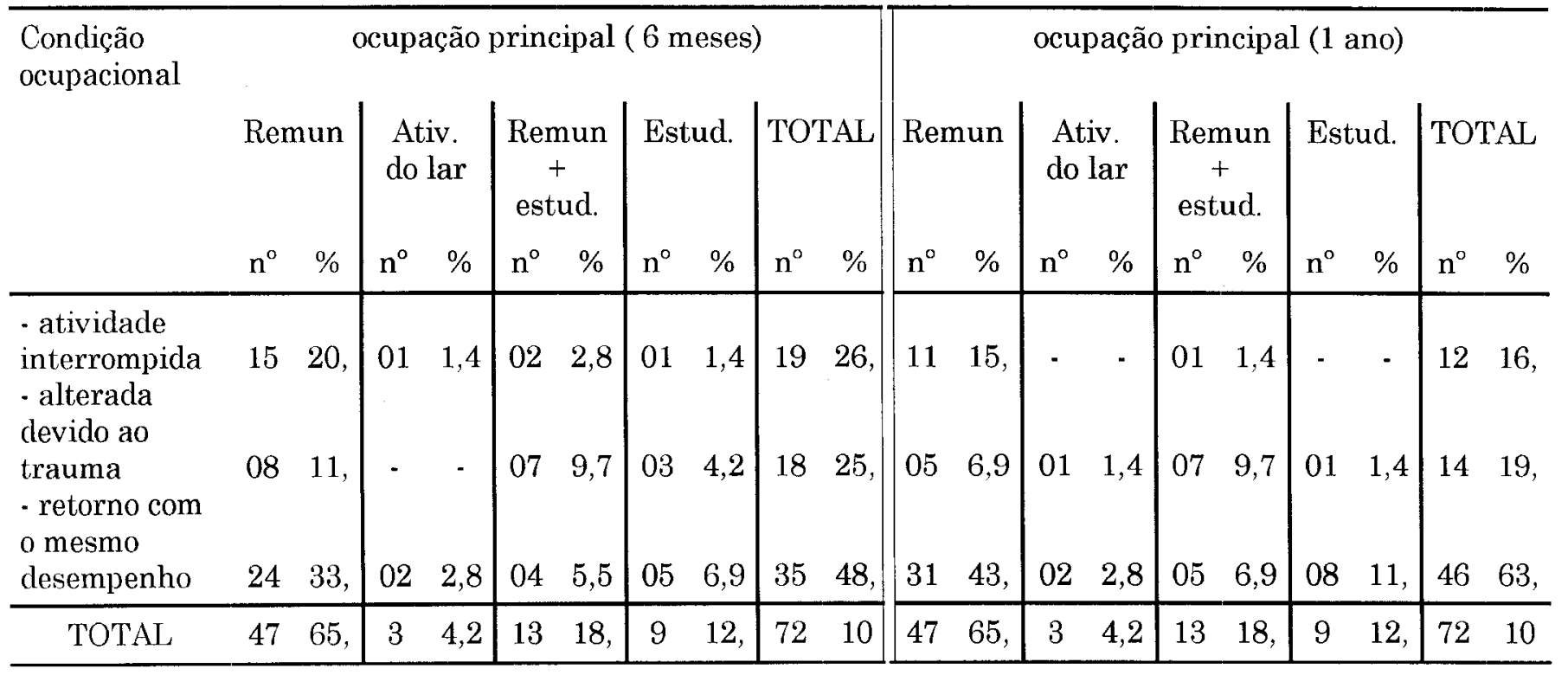

No geral, o percentual de vítimas que retornou à produtividade aos 6 meses foi de $73,6 \%(25,0 \%+$ $48,6 \%)$ e aos 12 meses de $83,3 \%(19,4 \%+63,9 \%)$ quando vítimas que retornaram a ocupação principal mantendo o mesmo nível de desempenho ou com limitações, foram consideradas produtivas.

Resultado semelhante foi encontrado por MACLENZIE et al $^{19}$ no seguimento de vítimas de trauma em geral. Eles verificaram que após 1 ano da alta hospitalar, $17,0 \%$ dos traumatizados em seguimento ainda encontravam-se incapazes de trabalhar, ir a escola ou realizar as atividades do lar. Resultados menos favoráveis foram obtidos por MORRIS et $\mathrm{al}^{22}$ quando analisam vítimas de trauma cujo custo da internação superou a 100.000 dólares. Nesses indivíduos o retorno à produtividade, avaliado pelo menos 1 ano pós-trauma, era de 54,5\%. Entretanto, nesse mesmo estudo, ao se analisarem separadamente as vítimas de TCE observou-se que o retorno à ocupação principal foi de $72,0 \%$.

No período de 1 ano, todas as pessoas envolvidas em atividades do lar ou somente estudantes antes do trauma haviam retornado à produtividade. O mesmo não ocorreu com os indivíduos que mantinham atividades remuneradas. Dos 13 estudantes que trabalhavam, 1 continuava afastado e das 47 vítimas com trabalho remunerado 11 continuavam com atividade interrompida, atingindo assim proporção de aproximadamente 1 afastado para cada 4 indivíduos.

Ainda que o período de 1 ano possa ser considerado suficiente para pelo menos iniciar o preparo dos afastados do trabalho remunerado para outra função compatível com sua incapacidade ${ }^{18}$ nenhuma das 12 vítimas que continuavam afastadas na FASE 2, encontrava-se em tal situação. Além disso, 2 vítimas, que não tinham possibilidade de retorno à sua função anterior, queixavam-se também de ausência de apoio social para preparo em outra área de atuação. Outro sentimento expresso pelos afastados era a vontade de retornar ao trabalho, contrapondo-se ao receio de desemprego em conseqüência da incapacidade de manter o mesmo nível de desempenho em sua função.

Considerando-se todos indivíduos que mantinham atividade remunerada, isto é, estudantes ou não que apresentavam esse tipo de ocupação, tinha-se 60 vítimas. Destas, no período de 1 ano, 12 não haviam retornado ao seu trabalho remunerado, perfazendo percentual de $20,0 \%$

Em estudos que analisam separadamente o retorno à atividade remunerada no período apos trauma, observa-se que resultados diferenciados das vítimas de TCE são apresentados quando o retorno aquela atividade e avaliada. RHODES et al ${ }^{25}$ verificaram que $75,3 \%$ dos indivíduos, atendidos em centro de trauma, retornaram a sua situação anterior de trabalho remunerado entre 6 meses e 2 anos e meio após o acidente ou violência, e que $46 \%$ dos desempregados inicialmente retornaram ao trabalho remunerado num período subseqüente. FRUTIGER et al $^{\text {? }}$ apresentam resultados semelhantes, avaliando traumatizados apos 5 anos de trauma. Estes autores especificam, entretanto, que dos $79 \%$ dos pacientes que se encontravam trabalhando, 11\% tiveram necessidade de mudar de ocupação e $35 \%$ referiram significantes problemas no trabalho. Ressaltam ainda que o desemprego é 
praticamente inexistente na Suíça, local onde foi realizado o estudo, e mesmo pessoas que são incapazes, dificilmente sofrem o risco de não conseguir emprego. Mesmo as vítimas de trauma grave, com valores do ISS em média de 39 e com lesões em pelo menos quatro regiões do corpo analisadas por KIVIOJA et al ${ }^{16}$ apresentaram níveis de retorno ao trabalho de $72 \%$ quando examinados entre 5 e 20 anos após o trauma. MACKENZIE et al $^{19}$ estudando os resultados do trauma e fatores relacionados a recuperação, observaram que $57 \%$ dos indivíduos que estavam trabalhando em tempo integral, antes do trauma, reassumiram seu emprego da, mesma forma, dentro de 1 ano. Entretanto, observaram também que as vítimas de TCE ou lesão medular grave são aquelas que apresentam o mais alto risco de não retornarem ao trabalho remunerado.

Corroborando tais resultados, uma capacidade bem menor de retorno ao emprego e encontrada especificamente nos estudos referentes às vítimas de TCE. TATE et al ${ }^{29}$ analisando conseqüências psicossociais do trauma craniano grave, observaram que um quinto da série de pacientes avaliados encontravam-se normalmente empregados após 3 anos de TCE e somente $40 \%$ dos indivíduos do grupo que apresentaram boa recuperação mantinham emprego nesse período. GENSEMER et $a^{10}$, acompanhando vítimas de TCE com aparente boa recuperação, mas com queixas cognitivas e de mudança de personalidade, apresentam resultados semelhantes, encontrando $65 \%$ da sua população desempregada e $35 \%$ trabalhando. RAPPAPORT et $\mathrm{al}^{24}$, analisando os resultados do TCE entre 5 e 10 anos apos sua ocorrência, verificaram que somente $11 \%$ dos indivíduos entrevistados consideravam-se capazes de manter um emprego sem modificações que atendessem suas limitações. Observaram ainda, que $61 \%$ das vítimas de TCE entrevistadas, encontravam-se desempregadas na época da entrevista, enquanto antes do trauma todas se encontravam empregadas. Aproxima-se dos resultados de RAPPAPORT et $\mathrm{al}^{24}$ aqueles indicados por GROSWASSER; SAZBON ${ }^{12}$. Neste último são incluídas vítimas de TCE que estiveram inconscientes por mais de 30 dias, mas que recuperaram a consciência. Observa-se em seus resultados, baseados em informações coletadas pelo menos um ano após alta do hospital de reabilitação, que somente $11,1 \%$ das vítimas avaliadas eram capazes de reassumir trabalho em mercado aberto e $48,6 \%$ eram incluídas em empregos especiais. Analisando material referente a trauma craniano do banco de dados da Universidade da Virginia, JANE; RIMEL ${ }^{14}$ descrevem que não houve retorno ao trabalho até 3 meses pós-trauma nos indivíduos com TCE grave; naqueles com TCE moderado o retorno foi de $33 \%$ e no grupo de TCE leve de $66 \%$. Por outro lado, ODDY et al $^{23}$, analisando retorno ao trabalho, 2 e 7 anos após TCE grave, verificaram que aqueles que eram desempregados 2 anos apos a injúria mantinham a mesma condição 7 anos após o trauma.

Melhores resultados de retorno ao trabalho remunerado são apresentados no presente estudo, quando comparados com publicações direcionadas especificamente a vítimas de TCE. Nessas pesquisas, eram diferentes a gravidade do trauma das vítimas e o período em que avaliações foram realizadas. Há portanto, perspectivas distintas na comparação desses resultados, quando se considera que no presente estudo vítimas de todas as gravidades foram analisadas, enquanto a maioria das incluídas nos estudos comentados são pessoas gravemente traumatizadas, grupo que tem atingido cerca de $10,0 \%$ daqueles internados por $\mathrm{TCE}^{17}$.

Ainda que os 6 primeiros meses possa ser considerado como intervalo de tempo de estabilização da recuperação do traumatizado e uma época em que a maioria das vítimas retorna às atividades que exercia anteriormente ao trauma, deficiências ou incapacidades residuais permanecem por períodos superiores a 1 ano as quais interferem na capacidade da vítima exercer papéis e funções que dela são esperados.

Embora o evento traumático geralmente leve somente segundos para ocorrer, seus efeitos permanecem por longos períodos na vítima e seus familiares, sendo poucos eventos na vida, que como o TCE, podem precipitar mudanças tão acentuadas em papéis, relações e objetivos de suas vítimas ${ }^{30}$

A necessidade de assistência desses traumatizados permanece, portanto, por longos períodos após TCE, e vão além da necessidade de auxílio para retomar suas capacidades funcionais. Controle dos riscos em potencial e da sintomatologia apresentada, suporte para reinserção social à medida que dificuldades se estabeleçam, apoio para estabilização emocional de indivíduo e família e obtenção de informações factuais são importantes demandas de assistência apresentada pelos traumatizados as quais deveriam ser atendidas com recursos e por equipe multiprofissional de saúde preparada para esse atendimento $0^{3,30}$. A ausência de tais condições de assistência, para o atendimento de muitas vítimas traumatizadas, conduz a maior dimensão do problema, tanto a indivíduos e famílias, como aumentam a magnitude das conseqüências do TCE na sociedade.

De fato, o TCE afeta a vítima pessoalmente, interpessoalmente e o seu sistema social. Além disso, seu impacto financeiro está duplamente presente. Por um lado, pelo custo hospitalar seguido de longo 
período de necessidade de suporte do sistema de saúde para reabilitação e por outro, compondo esse impacto estão as semanas e anos de perda de produtividade. Claramente, as ocorrências do TCE e suas conseqüências têm atingido proporções epidêmicas justificando atenção como um grande problema de saúde da sociedade.

\section{Fatores Relacionados ao Retorno ao Trabalho Remunerado}

No presente estudo, para analisar o retorno ao trabalho remunerado e as possíveis associações com o nível de escolaridade, apresenta-se na Figura 1 a distribuição das 60 vítimas que mantinham atividade remunerada no período anterior ao trauma, segundo seu nível de escolaridade e retorno à ocupação remunerada, tanto no período de 6 como de 12 meses pós-trauma.

Figura 1 - Distribuição das vítimas com atividade remunerada segundo escolaridade e retorno ao trabalho aos 6 e 12 meses pós-trauma. São Paulo, 1993/94.

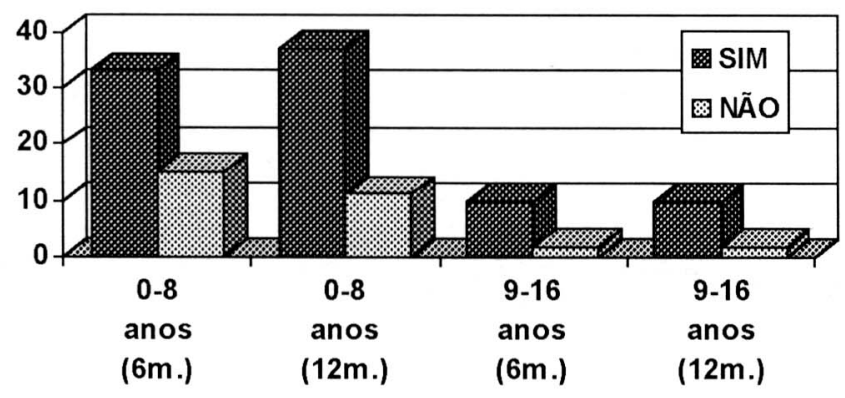

$0-8(6 \mathrm{~m})=$. escolaridade de 0 a 8 anos $(6$ meses pós-trauma $)$
$0-8(12 \mathrm{~m})=$. escolaridade de 0 a 8 anos $(12$ meses pós-trauma $)$
$9-16(6 \mathrm{~m})=$. escolaridade de 9 a 16 anos $(6$ meses pós-trauma $)$
$9-16(12 \mathrm{~m})=$. escolaridade de 9 a 16 anos $(12$ meses pós-trauma $)$

Como mostra a Figura 1, o retorno ao trabalho rem un erado das vítim as com escolaridade $\geq 9$ anos foi semelhante entre os 2 períodos de avaliação. No grupo com escolaridade $\leq$ a 8 anos o número de indivíduos afastados de sua atividade remunerada era aos 6 meses aproximadamente metade das vítimas, entretanto aos 12 meses tal proporção é reduzida a menos que $1 / 3$.

Para determinar a significância da diferença entre os grupos com escolaridade menor ou igual e maior que 8 anos, aplicou-se a prova exata de Fisher. Ainda que proporcionalmente pareça haver comportamento diferente entre os grupos quanto ao retorno ao trabalho a diferença observada não foi estatisticamente significativa.

Tais resultados diferem dos de outros estudos que mostram menor freqüência de retorno ao trabalho em vítimas de trauma com mais baixa escolaridade ${ }^{11,18,27}$. Entretanto, é interessante mais uma vez mencionar que o presente estudo englobou vítimas de TCE de diversas gravidades, enquanto nas publicações mencionadas, os participantes eram vítimas de trauma em geral ou de TCE leve.
Por outro lado, resultados do presente estudo são concordantes com o de RIMEL et al ${ }^{28}$ que analisam vítimas de TCE moderado e verificam que o retorno ao trabalho não foi significativamente associado com o nível de escolaridade. MORRIS et al ${ }^{22}$ também concluíram que escolaridade está entre os fatores que estatísticamente não indicam baixa probabilidade de retorno ao trabalho nas vítimas de trauma.

Outro fator que tem sido apontado como relacionado com retorno ao trabalho é o tipo de ocupação. Alguns estudos têm mostrado que indivíduos envolvidos em ocupações tipo "blue collar" têm menos retorno ao trabalho que os demais ${ }^{18,19,24}$. $\mathrm{RIMEL}^{26}$, referindo-se a trabalhadores envolvido neste tipo de ocupação, descreve estes indivíduos como aqueles que exercem ofícios instrumentais e semi-instrumentais.

Pensando-se numa provável similaridade, classificou-se inicialmente as ocupações conforme FONSECA ${ }^{6}$ em manuais não especializadas, manuais especilaizadas, sendo que as demais ocupações foram categorizadas como outras. 
Os critérios para essa categorização estão apresentados no ANEXO I, assim como a relação de ocupações considerada em cada uma das categorias.

Designou-se de Grupo A, aquele que incluía individuos com ocupações manuais não especilalizadas, GrupoB, manuais especializadas e Grupo C, outras ocupações. Pelo teste Qui-quadrado não se observou associação entre cada grupo de ocupação e o retorno ao trabalho remunerado quando testados isoladamente.

Figura 2 - Distribuição das vítimas com atividade remunerada segundo tipo de ocupação e retorno ao trabalho 6 e 12 meses pós-trauma. São Paulo, 1993/94.

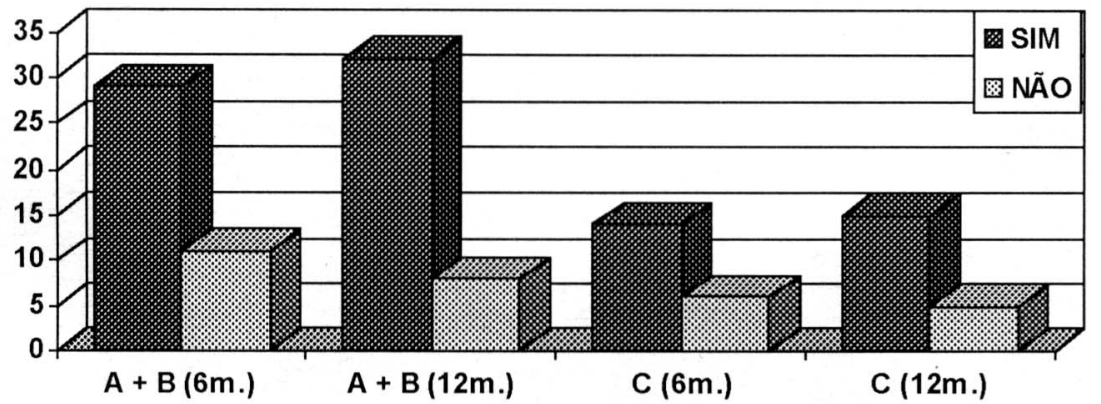

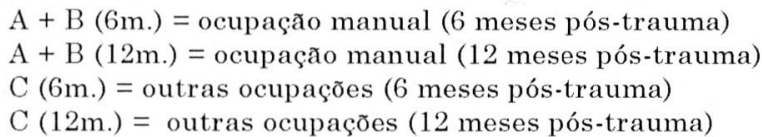

Observa-se na Figura 2 que aos 6 meses póstrauma o retorno ao trabalho ocorreu de forma semelhante nesses dois grupos, sendo entretanto no grupo de vítimas com ocupação manual proporcionalmente maior o contingente dos que retornaram ao trabalho no período de 1 ano, se comparado àqueles de outras ocupações. Assim como nível de escolaridade, o tipo de ocupação não foi um fator relacionado significativamente ao retorno ao trabalho. Tais resultados foram observados na aplicação da Prova exata de Fisher utilizada para determinar a significância de diferenças entre os grupos reunidos na combinação graficamente apresentada.

Resultados contraditórios foram observados por outros autores. O tipo de ocupação foi associada com retorno ao trabalho nos estudos de MACKENZIE et al ${ }^{18,19}$ e de RIMEL et al ${ }^{27}$ quando analisaram vítimas de TCE leve. Em outros estudos tal associação não foi observada ${ }^{13,22,28}$. É possível que diferentes períodos de avaliação pós-traumática, diferenças sócio-econômicas e culturais, assim como níveis ocupacionais desiguais tenham ocasionado essas diferenças.
Considerando-se entretanto que ocupações "blue collar" incluem ocupações manuais de forma genérica, outra análise dos dados foi realizada. $\mathrm{Na}$ Figura 2, estão apresentadas de forma agrupada as vítimas com ocupações manuais especializadas e não especializadas as quais são analisadas segundo seu retorno ao trabalho em comparação com aquelas com outros tipos de ocupação. 
de afastamento e a quantidade de pessoas que retornam ao trabalho apos o trauma, embora os resultados obtidos não apresentem evidências consistentes a esse respeito.

\section{CONCLUSÕES}

O estudo das vítimas de TCE com diferentes níveis de gravidade do trauma, diferentes níveis de alteração de consciência na primeira avaliação feita pelo neurocirurgião e idade entre 12 e 60 anos permitiu as conclusões enunciadas a seguir.

\section{Quanto ao retorno à produtividade}

- Aos 6 meses: A maioria (73,6\%) dos traumatizados já havia retornado à produtividade, havendo entretanto entre eles, $25,0 \%$ que apresentavam em conseqüência do trauma alterações em sua ocupação principal.

- 1 ano apos TCE: Retorno à produtividade ocorreu numa freqüência de $83,3 \%$ e destes, $19,4 \%$ tinham alterado a sua ocupação principal.

- Entre 6 meses e 1 ano:. Alterações favoráveis na produtividade das vítimas ocorreram nesse período tanto pelo aumento da freqüência de indivíduos que retornaram a produtividade, como pela diminuição daqueles que alteraram sua atividade.

Quanto ao retorno ao trabalho remunerado e as variáveis níveis de escolaridade e tipo de ocupação anterior ao trauma, não houve associação estatisticamente significativa, seja aos 6 meses como 1 ano após o evento traumático.

\section{REFERÊNCIAS BIBLIOGRÁFICAS}

THE ABBREVIATED Injury Scale : 1990 revision. Des Plaines, Association for the Advancement of Automotive Medicine, 1990

BAXT, W.G.: MOODY, P. The differential survival of trauma patients. J Trauma, v.27, n.6, p.602-6, 1987

CAMPBELL,C.H. Needs of relatives and helpfulness of support groups in severe head injury. Rehabil Nurs, v.13, n.6, p.320-5, 1988 .

DACEY, R. et al. Relative effects of brain and non-brain injuries on neuropsychological and psychosocial outcome. J Trauma, v.31, n.2, p.217-22, 1991.

DALOSSI,T.; KOIZUMI,M.S. Estudo comparativo da gravidade do trauma de pacientes com ou sem traumatismo cranioencefálico. Rev Bras Neurol, v.30, n.6, p.181-9, 1994.

FONSECA,G.T,da Modelo para uma classificação de ocupaçoes. Rev Bras Est Pedag., v.47, n.106, p.274-312, 1967.

FRUTIGER, A et al. Five years' follow-up of severely injured ICU patients. J.Trauma, v.31, n.9, p.1216-26, 1991

FUNDAÇÃO SISTEMA ESTADUAL DE ANÁLISES DE DADOS - SEADE. Taxa de desemprego na grande São Paulo em 1993. São Paulo, s.d. /Mimeografado/.
GENNARELLI, T.A. et al. Mortality of patients with head injury and extracranial injury treated in trauma centers. J Trauma, v.29, n.9, p.1193-202, 1989 .

GENSEMER, I.B. et al. Behavioral consequences of trauma. J Trauma, v.28, n.1, p.44-9, 1988.

GLANCY, K.E. et al. A study of recovery in trauma patients J Trauma, v.33, n.4, p.602-9, 1992.

GROSWASSER, Z.; SAZBON, L. Outcome in 134 patients with prolonged posttraumatic unawareness. J Neurosurg, v.72 n.1, p.81-4, 1990 .

HOLBROOK,T.L. et al. Functional limitation after major trauma: a more sensitive assessment using the Quality of Well-being Scale - the Trauma recovery pilot project. J Trauma, v.36, n.1, p.74-8, 1994.

JANE, J.A.; RIMEL, R.W. Prognosis in head injury. Clin Neurosurg, v.29, p.346-52, 1982.

JENNETT, B. et al. Severe head injuries in three countries. J.Neurol Neurosurg Psychiatry,

v.40, n.3, p.291-8, 1977.

KIVIOJA, A.H. et al. Is the treatment of the most severe multiply injured patients worth the effort? - a follow up examination 5 to 20 years after severe multiple injury. J Trauma, v.30, n.4, p.480-3, 1990.

KRAUS, J.F. Epidemiology of head injury. In: COOPER, P.R Head injury, 3.ed. New York, Williams \& Wilkins, 1993. cap.1, p.1-25.

MACKENZIE, E.J. et al. Factors influencing return to work following hospitalization for traumatic injury. Am J Public Health, v.77, n.3, p.329-34, 1987

MACKENZIE, E.J. et al. Functional recovery and medical costs of trauma: an analysis by type and severity of injury. J Trauma, v.28, n.3, p.281-97, 1988

MANREZA, L.A.; RIBAS, G.C. Traumatisinos craniencefálicos. In NITRINI, R.; BACHESCHI, L.A. A Neurologia que todo médico deve saber. São Paulo, Maltese, 1991 cap.8, p.149-66.

MOCK, C.N. et al. Trauma outcomes in the rural developing world: comparison with an urban level I trauma center. J Trauma, v. $35, \mathrm{n} .4, \mathrm{p} .518-23,1993$.

MORRIS, J.A. et al. Trauma patients return to productivity. J Trauma, v.31, n.6, p.827-34, 1991.

ODDY, M. et al. Social adjustment after closed head injury: a further follow-up seven years after injury. J Neurol Neurosurg Psychiatry, v.48, n. 6, p.564-8, 1985.

RAPPAPORT, M. et al. Head injury outcome up to ten years later. Arch Phys Med Rehabil, v.70, n.13, p.885-92, 1989.

RHODES, M. et al. Quality of life after the trauma center. J.Trauma, v.28, n.7, p.931-8, 1988.

RIMEL, R.W. A prospective study of patients with central nervous system trauna. J. Neurosurg Nurs, v.13, n.3, p.132-41, 1981 .

RIMEL, R.W. et al. Disability caused by minor head injury Neurosurgery, v.9, n.3, p.221-8, 1981 .

RIMEL, R.W. et al. Moderate head injury: completing the clinical spectrum of brain trauma. Neurosurgery, v.11, n.3 p.344-51, 1982.

TATE, R.L. et al. Psychosocial outcome for the survivors of severe blunt head injury: the results from a consecutive series of 100 patients. J.Neurol Neurosurg Psychiatry, v.52, n.10, p.1128-34, 1989 .

TESTANI-DUFOUR, L. et al. Traumatic brain injury: a familiy experience. J Neuroci Nurs, v.24, n.6, p.317-23, 1992. 


\section{ANEXO I}

\section{Tipos de ocupação antes do trauma.}

(Baseado nos níveis ocupacionais apresentados por FONSECA ${ }^{6}$ )

\section{A}

Ocupações manuais não especializadas: aquelas que envolvem trabalho manual e que não exigem experiência profissional prévia ou preparo profissional formal.

\section{B}

Ocupações manuais especializadas: aquelas que envolvem tarefas manuais que são precisas, minuciosas e conseqüentemente exigem elevado grau de habilidade manual. Ao contrário das ocupações manuais não especializadas, exigem experiência profissional anterior ou preparo formal.

\section{C}

Outras ocupações: aquelas que envolvem tarefas não manuais que exigem diferentes graus de especialização e portanto, exigem níveis mínimos de escolaridade e/ou experiência profissional anterior. Inclui cargos de supervisão, de gerência e técnico de nível médio.

\begin{tabular}{|c|c|c|}
\hline $\begin{array}{l}\text { Ocupações incluídas na categoria } \\
\text { A: }\end{array}$ & $\begin{array}{l}\text { Ocupações incluídas na categoria } \\
\text { B: }\end{array}$ & $\begin{array}{l}\text { Ocupações incluídas na categoria } \\
\text { C: }\end{array}$ \\
\hline $\begin{array}{l}\text { repositor de mercadoria em } \\
\text { supermercado; } \\
\text {. faxineiro e vigilante; } \\
\text { lavadeira; } \\
\text {. ajudante de motorista; } \\
\text { ajudante geral (fábrica de } \\
\text { margarina); } \\
\text { ajudante em metalúrgica; } \\
\text { lombador (carregador de carne); } \\
\text { ajudante de montagem de } \\
\text { "stand"; } \\
\text { ajudante geral de construção } \\
\text { civil; } \\
\text { empregada doméstica; } \\
\text { - montador em linha de produção } \\
\text { (plástico); } \\
\text {. segurança; } \\
\text { servente de pedreiro; } \\
\text {. borracheiro (empresa de ônibus); } \\
\text { faxineiro; } \\
\text { vigia; } \\
\text {. caseiro; } \\
\text {. auxiliar de embalagem; } \\
\text { ajudante geral (casas } \\
\text { pré-moldadas); } \\
\text { office-boy; } \\
\text { balconista de padaria. }\end{array}$ & $\begin{array}{l}\text {. artesão em metal; } \\
\text { meio oficial de manutenção; } \\
\text { massagista e caldereiro em } \\
\text { sauna; } \\
\text {. mecânico de retífica de motor; } \\
\text { - pedreiro; } \\
\text {. mecânico de manutenção; } \\
\text { cabeleireiro; } \\
\text {. cozinheira; } \\
\text {. colocador de papel de parede; } \\
\text {. costureira; } \\
\text {. taxista; } \\
\text { operador de funilaria industrial e } \\
\text { caldereiro; } \\
\text {. jogador de futebol júnior; } \\
\text { técnico de exaustores; } \\
\text {. mecânico de produção; } \\
\text { reparos gerais (pintor, } \\
\text { carpinteiro, encanador); } \\
\text { funileiro; } \\
\text { bombeiro. }\end{array}$ & $\begin{array}{l}\text {. assistente de faturamento; } \\
\text { comerciante; } \\
\text {. decorador; } \\
\text {. empreiteiro; } \\
\text {. assistente financeiro; } \\
\text {. vendedor de loja de móveis; } \\
\text {. conferente (restaurante); } \\
\text {. caixa de banco; } \\
\text { vendedor de anúncio de jornal e } \\
\text { fotógrafo; } \\
\text {. controlador operacional da } \\
\text { CETESB; } \\
\text {. encarregado geral em loja de } \\
\text { material de construção; } \\
\text { professor; } \\
\text {. chefe de secção em metalúrgica; } \\
\text {. cobrador de ônibus; } \\
\text {. supervisor técnico em } \\
\text { metalúrgica; } \\
\text { fiscal de ônibus; } \\
\text { proprietário de lavanderia } \\
\text { industrial; } \\
\text { gerente operacional de empresa } \\
\text { de transportes; } \\
\text {. vendedor de auto-peças. }\end{array}$ \\
\hline
\end{tabular}

